\title{
Unruh effect in quantum information beyond the single-mode approximation
}

\author{
David E. Bruschi, ${ }^{1}$ Jorma Louko, ${ }^{1}$ Eduardo Martín-Martínez, ${ }^{2}$ Andrzej Dragan, ${ }^{3}$ and Ivette Fuentes ${ }^{1, *}$ \\ ${ }^{1}$ School of Mathematical Sciences, University of Nottingham, Nottingham NG7 2RD, United Kingdom \\ ${ }^{2}$ Instituto de Física Fundamental, Consejo Superior de Investigaciones Científicas, Serrano 113-B, E-28006 Madrid, Spain \\ ${ }^{3}$ Institute of Theoretical Physics, University of Warsaw, Hoża 69, PL-00-049 Warsaw, Poland
}

(Received 27 July 2010; published 26 October 2010)

\begin{abstract}
We address the validity of the single-mode approximation that is commonly invoked in the analysis of entanglement in noninertial frames and in other relativistic quantum-information scenarios. We show that the single-mode approximation is not valid for arbitrary states, finding corrections to previous studies beyond such approximations in the bosonic and fermionic cases. We also exhibit a class of wave packets for which the single-mode approximation is justified subject to the peaking constraints set by an appropriate Fourier transform.

DOI: 10.1103/PhysRevA.82.042332

PACS number(s): 03.67.Mn, 03.65.Yz, 04.62.+v
\end{abstract}

\section{INTRODUCTION}

The question of understanding entanglement in noninertial frames has been central to the development of the emerging field of relativistic quantum information [1-20]. The main aim of this field is to incorporate relativistic effects to improve quantum-information tasks (such as quantum teleportation) and to understand how such protocols would take place in curved space times. In most quantum-information protocols, entanglement plays a prominent role. Therefore, it is of great interest to understand how it can be degraded [4,5,20-22] or created [23-25] by the presence of horizons or space-time dynamics.

Previous analyses show that entanglement between modes of bosonic or fermionic fields is degraded from the perspective of observers moving in uniform acceleration. In this paper, we analyze the validity of the single-mode approximation commonly used in such analyses and show that the approximation is justified only for a special family of states. The single-mode approximation, which was introduced in Refs. [1,12] has been extensively used in the literature not only in discussions concerning entanglement, but also in other relativistic quantuminformation scenarios [4,5,15,17,19,21,22,26-33]. A deeper understanding of how the Unruh effect degrades entanglement is of crucial importance not only for fundamental questions, but also to engineer a practical method to experimentally detect such an effect. So far, not only has the effect not been measured, but also its very existence has been subject to some controversy (see, for instance, Refs. [34-36]).

In the canonical scenario considered in the study of entanglement in noninertial frames, the field, from the inertial perspective, is considered to be in a state where all modes are in the vacuum state except for two of them, which are in a two-mode entangled state. For example, the Bell state,

$$
|\Psi\rangle_{\mathrm{M}}=\frac{1}{\sqrt{2}}\left(\left|0_{\omega}\right\rangle_{\mathrm{M}}\left|0_{\omega^{\prime}}\right\rangle_{\mathrm{M}}+\left|1_{\omega}\right\rangle_{\mathrm{M}}\left|1_{\omega^{\prime}}\right\rangle_{\mathrm{M}}\right),
$$

where M labels Minkowski states and $\omega, \omega^{\prime}$ are two Minkowski frequencies. Two inertial observers, Alice and Bob, each carrying a monochromatic detector sensitive to frequencies $\omega$ and $\omega^{\prime}$, respectively, would find maximal correlations

\footnotetext{
*Previously known as Fuentes-Guridi and Fuentes-Schuller.
}

in their measurements, since the Bell state is maximally entangled. It is then interesting to investigate to what degree the state is entangled when described by observers in uniform acceleration. In the simplest scenario, Alice is again considered to be inertial, and a uniformly accelerated observer Rob is introduced, who carries a monochromatic detector sensitive to mode $\omega^{\prime}$. To study this situation, the states corresponding to Rob must be transformed into the appropriate basis, in this case, the Rindler basis. It is then when the single-mode approximation is invoked to relate Minkowski single-particle states $\left|1_{\omega^{\prime}}\right\rangle_{M}$ to states in Rindler space.

We argue that the single-mode approximation is not valid for general states. However, the approximation holds for a family of peaked Minkowski wave packets provided constraints imposed by an appropriate Fourier transform are satisfied. We show that the state analyzed canonically in the literature corresponds to an entangled state between a Minkowski and a special type of Unruh mode. Therefore, we revise previous results for both bosonic- and fermionic-field entanglement. The results are qualitatively similar to those obtained under the single-mode approximation. We confirm that entanglement is degraded with acceleration, vanishing in the infinite-acceleration limit in the bosonic case and reaching a nonvanishing minimum for fermionic fields. However, we find that, in the fermionic case, the degree to which entanglement is degraded depends on the election of Unruh modes.

The paper is organized as follows: In Sec. II, we introduce the transformations between Minkowski, Unruh, and Rindler modes. In Sec. III, we analyze the entanglement degradation due to the Unruh effect for scalar fields including corrections to the single-mode approximation. We exhibit, in Sec. IV, states for which the single-mode approximation is justified in the massless and massive bosonic cases. In Sec. V, the degradation of entanglement between fermionic modes is addressed. Finally, conclusions and discussions are presented in Sec. VI.

\section{MINKOWSKI, UNRUH, AND RINDLER MODES}

We consider a real massless scalar field $\phi$ in a twodimensional Minkowski space time. The field equation is the massless Klein-Gordon equation, $\square \phi=0$. The (indefinite) 
Klein-Gordon inner product reads

$$
\left(\phi_{1}, \phi_{2}\right)=i \int_{\Sigma} \phi_{1}^{*} \stackrel{\leftrightarrow}{\partial_{a}} \phi_{2} n^{a} d \Sigma,
$$

where $n^{a}$ is a future-pointing normal vector to the spacelike hypersurface $\Sigma$ and $d \Sigma$ is the volume element on $\Sigma$.

The Klein-Gordon equation can be solved in Minkowski coordinates $(t, x)$, which are an appropriate choice for inertial observers. The positive-energy mode solutions with respect to the timelike Killing vector field $\partial_{t}$ are given by

$$
u_{\omega, \mathrm{M}}(t, x)=\frac{1}{\sqrt{4 \pi \omega}} \exp [-i \omega(t-\epsilon x)],
$$

where $\omega>0$ is the Minkowski frequency and the discrete index $\epsilon$ takes the value 1 for modes with positive momentum (the right movers) and the value -1 for modes with negative momentum (the left movers). As the right movers and the left movers decouple, we have suppressed the index $\epsilon$ on the left-hand side of Eq. (3), and we continue to do so in all the formulas. The mode solutions and their complex conjugates are normalized in the usual sense of Dirac $\delta$ functions in $\omega$ as

$$
\begin{gathered}
\left(u_{\omega, \mathrm{M}}, u_{\omega^{\prime}, \mathrm{M}}\right)=\delta_{\omega \omega^{\prime}}, \\
\left(u_{\omega, \mathrm{M}}^{*}, u_{\omega^{\prime}, \mathrm{M}}^{*}\right)=-\delta_{\omega \omega^{\prime}}, \\
\left(u_{\omega, \mathrm{M}}^{*}, u_{\omega^{\prime}, \mathrm{M}}\right)=0 .
\end{gathered}
$$

The Klein-Gordon equation can also be separated in coordinates that are adapted to the Rindler family of uniformly accelerated observers. Let region I (respectively, region II) denote the wedge $|t|<x(x<-|t|)$. In each of the wedges, we introduce the Rindler coordinates $(\eta, \chi)$ by [37]

$$
\eta=\arctan \left(\frac{t}{x}\right), \quad \chi=\sqrt{x^{2}-t^{2}},
$$

where $0<\chi<\infty$ and $-\infty<\eta<\infty$ individually in each wedge. The curve $\chi=1 / a$, where $a$ is a positive constant of dimension inverse length, is then the world line of a uniformly accelerated observer whose proper acceleration equals $a$, and the proper time for this observer is given by $\eta / a$ in I and by $-\eta / a$ in II. (See Fig. 1.) Note that $\partial_{\eta}$ is a timelike Killing vector in both I and II, and it is future pointing in I but past pointing in II.

Separating the Klein-Gordon equation in regions I and II in the Rindler coordinates yields the solutions,

$$
\begin{aligned}
& u_{\Omega, \mathrm{I}}(t, x)=\frac{1}{\sqrt{4 \pi \Omega}}\left(\frac{x-\epsilon t}{l_{\Omega}}\right)^{i \epsilon \Omega}, \\
& u_{\Omega, \mathrm{II}}(t, x)=\frac{1}{\sqrt{4 \pi \Omega}}\left(\frac{\epsilon t-x}{l_{\Omega}}\right)^{-i \epsilon \Omega},
\end{aligned}
$$

where $\epsilon=1$ again corresponds to right movers and $\epsilon=-1$ to left movers, $\Omega$ is a positive dimensionless constant, and $l_{\Omega}$ is a positive constant of dimension length. As $\partial_{\eta} u_{\Omega, \mathrm{I}}=$ $-i \Omega u_{\Omega, \mathrm{I}}$ and $\partial_{\eta} u_{\Omega, \mathrm{II}}=i \Omega u_{\Omega, \mathrm{II}}, u_{\Omega, \mathrm{I}}$ and $u_{\Omega, \mathrm{II}}$ are the positivefrequency mode functions with respect to the future-pointing Rindler-Killing vectors $\pm \partial_{\eta}$ in their respective wedges, and $\Omega$ is the dimensionless Rindler frequency. The dimensional

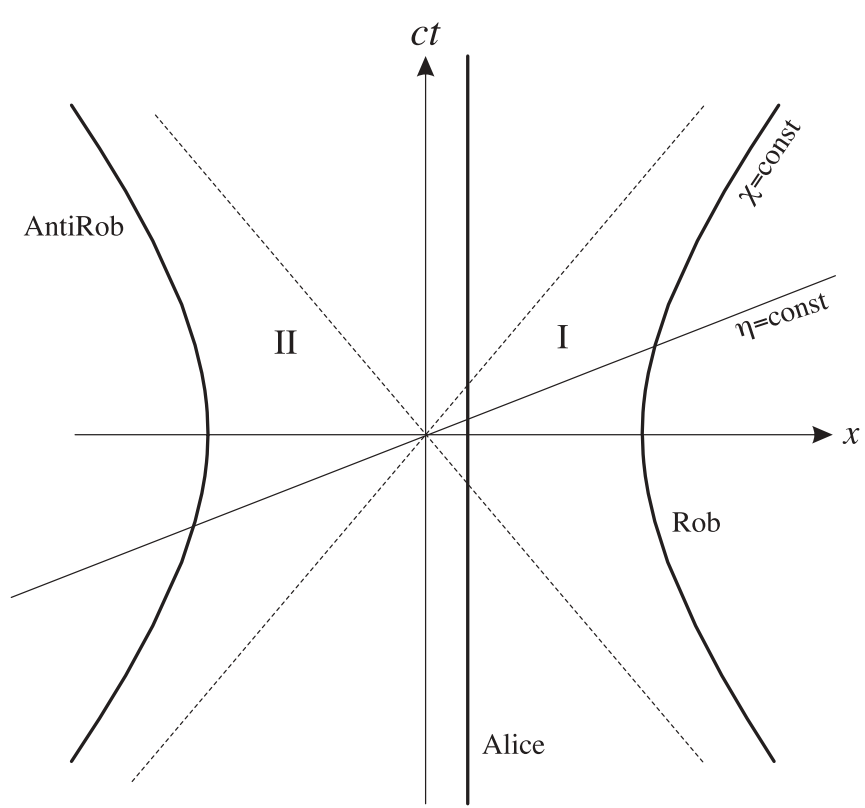

FIG. 1. Rindler space-time diagram: Lines of constant position $\chi=$ const are hyperbolae, and all curves of constant $\eta$ are straight lines that come from the origin. A uniformly accelerated observer Rob travels along a hyperbola constrained to either region I or region II.

frequency with respect to the proper time of a Rindler observer located at $\chi=1 / a$ is given in terms of the dimensionless $\Omega$ by $\Omega_{a}=a \Omega$. The modes are $\delta$ normalized in $\Omega$ in their respective wedges as usual.

Note that the choice of the constant $l_{\Omega}$ is equivalent to specifying the phase of the Rindler modes. Hence, this choice is purely a matter of convention, and it can be made independently for each $\Omega$ and $\epsilon$. We will shortly specify the choice so that the transformation between the Minkowski and the Rindler modes becomes simple.

A third basis of interesting solutions to the field equation is provided by the Unruh modes, defined by

$$
\begin{aligned}
& u_{\Omega, \mathrm{R}}=\cosh \left(r_{\Omega}\right) u_{\Omega, \mathrm{I}}+\sinh \left(r_{\Omega}\right) u_{\Omega, \mathrm{II}}^{*} \\
& u_{\Omega, \mathrm{L}}=\cosh \left(r_{\Omega}\right) u_{\Omega, \mathrm{II}}+\sinh \left(r_{\Omega}\right) u_{\Omega, \mathrm{I}}^{*}
\end{aligned}
$$

where $\tanh r_{\Omega}=e^{-\pi \Omega}$. While the Unruh modes have a sharp Rindler frequency, an analytic continuation argument shows that they are purely positive-frequency linear combinations of the Minkowski modes [38,39]. Hence, it is convenient to examine the transformation between the Minkowski and the Rindler modes in two stages:

(1) The well-known transformation (7) between the Unruh and the Rindler modes isolates the consequences of the differing Minkowski and the Rindler definitions of positive frequency.

(2) The less well-known transformation between the Minkowski and the Unruh modes [37] shows that a monochromatic wave in the Rindler basis corresponds to a nonmonochromatic superposition in the Minkowski basis.

It is these latter effects from which the new observations in this paper will stem. 
To find the Bogoliubov transformations that relate the bases, we expand the field in each of the bases as

$$
\begin{gathered}
\phi=\int_{0}^{\infty}\left(a_{\omega, \mathrm{M}} u_{\omega, \mathrm{M}}+a_{\omega, \mathrm{M}}^{\dagger} u_{\omega, \mathrm{M}}^{*}\right) d \omega \\
=\int_{0}^{\infty}\left(A_{\Omega, \mathrm{R}} u_{\Omega, \mathrm{R}}+A_{\Omega, \mathrm{R}}^{\dagger} u_{\Omega, \mathrm{R}}^{*}+A_{\Omega, \mathrm{L}} u_{\Omega, \mathrm{L}}+A_{\Omega, \mathrm{L}}^{\dagger} u_{\Omega, \mathrm{L}}^{*}\right) d \Omega \\
=\int_{0}^{\infty}\left(a_{\Omega, \mathrm{I}} u_{\Omega, \mathrm{I}}+a_{\Omega, \mathrm{I}}^{\dagger} u_{\Omega, \mathrm{I}}^{*}+a_{\Omega, \mathrm{II}} u_{\Omega, \mathrm{II}}+a_{\Omega, \mathrm{II}}^{\dagger} u_{\Omega, \mathrm{II}}^{*}\right) d \Omega,
\end{gathered}
$$

where $a_{\omega, \mathrm{M}}, A_{\Omega, \mathrm{R}}, A_{\Omega, \mathrm{L}}$, and $a_{\Omega, \mathrm{I}}, a_{\Omega, \mathrm{II}}$ are the Minkowski, Unruh, and Rindler annihilation operators, respectively. The usual bosonic commutation relations $\left[a_{\omega, \mathrm{M}}, a_{\omega^{\prime}, \mathrm{M}}^{\dagger}\right]=$ $\delta_{\omega \omega^{\prime}},\left[A_{\Omega, \mathrm{R}}, A_{\Omega^{\prime}, \mathrm{R}}^{\dagger}\right]=\left[A_{\Omega, \mathrm{L}}, A_{\Omega^{\prime}, \mathrm{L}}^{\dagger}\right]=\delta_{\Omega \Omega^{\prime}}$ and $\left[a_{\Omega, \mathrm{I}}, a_{\Omega^{\prime}, \mathrm{I}}^{\dagger}\right]=$ $\left[a_{\Omega, \mathrm{II}}, a_{\Omega^{\prime}, \mathrm{II}}^{\dagger}\right]=\delta_{\Omega \Omega^{\prime}}$ hold, and commutators for mixed R, L and mixed I, II vanish. The transformation between the Unruh and the Rindler bases is given by Eq. (7). The transformation between the Minkowski and the Unruh bases can be evaluated by taking appropriate inner products of formula (8) with the mode functions [37], with the result,

$$
\begin{gathered}
u_{\omega, \mathrm{M}}=\int_{0}^{\infty}\left(\alpha_{\omega \Omega}^{\mathrm{R}} u_{\Omega, \mathrm{R}}+\alpha_{\omega \Omega}^{\mathrm{L}} u_{\Omega, \mathrm{L}}\right) d \Omega, \\
u_{\Omega, \mathrm{R}}=\int_{0}^{\infty}\left(\alpha_{\omega \Omega}^{\mathrm{R}}\right)^{*} u_{\omega, \mathrm{M}} d \omega, \\
u_{\Omega, \mathrm{L}}=\int_{0}^{\infty}\left(\alpha_{\omega \Omega}^{\mathrm{L}}\right)^{*} u_{\omega, \mathrm{M}} d \omega
\end{gathered}
$$

where

$$
\begin{aligned}
& \alpha_{\omega \Omega}^{\mathrm{R}}=\frac{1}{\sqrt{2 \pi \omega}} \sqrt{\frac{\Omega \sinh \pi \Omega}{\pi}} \Gamma(-i \epsilon \Omega)\left(\omega l_{\Omega}\right)^{i \epsilon \Omega}, \\
& \alpha_{\omega \Omega}^{\mathrm{L}}=\frac{1}{\sqrt{2 \pi \omega}} \sqrt{\frac{\Omega \sinh \pi \Omega}{\pi}} \Gamma(i \epsilon \Omega)\left(\omega l_{\Omega}\right)^{-i \epsilon \Omega} .
\end{aligned}
$$

By the properties of the $\Gamma$ function (Ref. [40], formula 5.4.3), we can take advantage of the arbitrariness of the constants $l_{\Omega}$ and choose them so that Eq. (10) simplifies to

$$
\begin{aligned}
\alpha_{\omega \Omega}^{\mathrm{R}} & =\frac{1}{\sqrt{2 \pi \omega}}(\omega l)^{i \epsilon \Omega}, \\
\alpha_{\omega \Omega}^{\mathrm{L}} & =\frac{1}{\sqrt{2 \pi \omega}}(\omega l)^{-i \epsilon \Omega},
\end{aligned}
$$

where $l$ is an overall constant of dimension length, independent of $\epsilon$ and $\Omega$.

The transformations between the modes give rise to transformations between the corresponding field operators. From Eq. (9), the Minkowski and Unruh operators are related by

$$
\begin{gathered}
a_{\omega, \mathrm{M}}=\int_{0}^{\infty}\left[\left(\alpha_{\omega \Omega}^{\mathrm{R}}\right)^{*} A_{\Omega, \mathrm{R}}+\left(\alpha_{\omega \Omega}^{\mathrm{L}}\right)^{*} A_{\Omega, \mathrm{L}}\right] d \Omega, \\
A_{\Omega, \mathrm{R}}=\int_{0}^{\infty} \alpha_{\omega \Omega}^{\mathrm{R}} a_{\omega, \mathrm{M}} d \omega \\
A_{\Omega, \mathrm{L}}=\int_{0}^{\infty} \alpha_{\omega \Omega}^{\mathrm{L}} a_{\omega, \mathrm{M}} d \omega
\end{gathered}
$$

and from Eq. (7), the Unruh and Rindler operators are related by

$$
\begin{aligned}
& a_{\Omega, \mathrm{I}}=\cosh \left(r_{\Omega}\right) A_{\Omega, \mathrm{R}}+\sinh \left(r_{\Omega}\right) A_{\Omega, \mathrm{L}}^{\dagger}, \\
& a_{\Omega, \mathrm{II}}=\cosh \left(r_{\Omega}\right) A_{\Omega, \mathrm{L}}+\sinh \left(r_{\Omega}\right) A_{\Omega, \mathrm{R}}^{\dagger} .
\end{aligned}
$$

We can now investigate how the vacua and excited states defined with respect to the different bases are related. Since the transformation between the Minkowski and the Unruh bases does not mix the creation and annihilation operators, these two bases share the common Minkowski vacuum state $|0\rangle_{\mathrm{M}}=|0\rangle_{\mathrm{U}}=\prod_{\Omega}\left|0_{\Omega}\right\rangle_{\mathrm{U}}$, where $A_{\Omega, \mathrm{R}}\left|0_{\Omega}\right\rangle_{\mathrm{U}}=A_{\Omega, \mathrm{L}}\left|0_{\Omega}\right\rangle_{\mathrm{U}}=$ 0 . However, $|0\rangle_{\mathrm{U}}$ does not coincide with the Rindler vacuum: If one makes the ansatz,

$$
\left|0_{\Omega}\right\rangle_{\mathrm{U}}=\sum_{n} f_{\Omega}(n)\left|n_{\Omega}\right\rangle_{\mathrm{I}}\left|n_{\Omega}\right\rangle_{\mathrm{II}},
$$

where $\left|n_{\Omega}\right\rangle_{\mathrm{I}}$ is the state with $n$ Rindler I excitations over the Rindler I vacuum $\left|0_{\Omega}\right\rangle_{\mathrm{I}}$, and similarly, $\left|n_{\Omega}\right\rangle_{\mathrm{II}}$ is the state with $n$ Rindler II excitations over the Rindler II vacuum $\left|0_{\Omega}\right\rangle_{\mathrm{II}}$, use of Eq. (13) shows that the coefficient functions are given by $f_{\Omega}(n)=\tanh ^{n} r_{\Omega} / \cosh r_{\Omega}$. Thus, $|0\rangle_{\mathrm{U}}$ is a two-mode squeezed state of Rindler excitations over the Rindler vacuum for each $\Omega$.

Although states with a completely sharp value of $\Omega$ are not normalizable, we may approximate normalizable wave packets that are sufficiently narrowly peaked in $\Omega$ by taking a fixed $\Omega$ and renormalizing the Unruh and Rindler commutators to read $\left[A_{\Omega, \mathrm{R}}, A_{\Omega, \mathrm{R}}^{\dagger}\right]=\left[A_{\Omega, \mathrm{L}}, A_{\Omega, \mathrm{L}}^{\dagger}\right]=1$ and $\left[a_{\Omega, \mathrm{I}}, a_{\Omega, \mathrm{I}}^{\dagger}\right]=$ $\left[a_{\Omega, \mathrm{II}}, a_{\Omega, \mathrm{II}}^{\dagger}\right]=1$, with the commutators for mixed $\mathrm{R}, \mathrm{L}$ and mixed I, II vanishing. In this idealization of sharp peaking in $\Omega$, the most general creation operator that is of purely positive Minkowski frequency can be written as a linear combination of the two Unruh creation operators, in the form

$$
a_{\Omega, \mathrm{U}}^{\dagger}=q_{\mathrm{L}} A_{\Omega, \mathrm{L}}^{\dagger}+q_{\mathrm{R}} A_{\Omega, \mathrm{R}}^{\dagger},
$$

where $q_{\mathrm{R}}$ and $q_{\mathrm{L}}$ are complex numbers with $\left|q_{\mathrm{R}}\right|^{2}+\left|q_{\mathrm{L}}\right|^{2}=1$. Note that $\left[a_{\Omega, \mathrm{U}}, a_{\Omega, \mathrm{U}}^{\dagger}\right]=1$. From Eqs. (14) and (15), we then see that adding one idealized particle of this kind into Minkowski vacuum, of a purely positive Minkowski frequency, yields the state,

$$
\begin{gathered}
a_{\Omega, \mathrm{U}}^{\dagger}\left|0_{\Omega}\right\rangle_{\mathrm{U}}=\sum_{n=0}^{\infty} f_{\Omega}(n) \frac{\sqrt{n+1}}{\cosh r_{\Omega}}\left|\Phi_{\Omega}^{n}\right\rangle, \\
\left|\Phi_{\Omega}^{n}\right\rangle=q_{\mathrm{L}}\left|n_{\Omega}\right\rangle_{\mathrm{I}}\left|(n+1)_{\Omega}\right\rangle_{\mathrm{II}}+q_{\mathrm{R}}\left|(n+1)_{\Omega}\right\rangle_{\mathrm{I}}\left|n_{\Omega}\right\rangle_{\mathrm{II}} .
\end{gathered}
$$

In previous studies on relativistic quantum information, it has been common to consider a state of the form (16) with $q_{\mathrm{R}}=1$ and $q_{\mathrm{L}}=0$. The previous discussion shows that this choice for $q_{\mathrm{R}}$ and $q_{\mathrm{L}}$ is rather special; in particular, it breaks the symmetry between the right and left Rindler wedges. Next, we will address how entanglement is modified for these sharp $\Omega$ states when both $q_{\mathrm{R}}$ and $q_{\mathrm{L}}$ are present, and we then turn to examine the assumption of sharp $\Omega$.

\section{ENTANGLEMENT REVISED BEYOND THE SINGLE-MODE APPROXIMATION}

In the relativistic quantum-information literature, the single-mode approximation $a_{\omega, \mathrm{M}} \approx a_{\omega, \mathrm{U}}$ is considered to 
relate Minkowski and Unruh modes. The main argument for taking this approximation is that the distribution,

$$
a_{\omega, \mathrm{M}}=\int_{0}^{\infty}\left[\left(\alpha_{\omega \Omega}^{\mathrm{R}}\right)^{*} A_{\Omega, \mathrm{R}}+\left(\alpha_{\omega \Omega}^{\mathrm{L}}\right)^{*} A_{\Omega, \mathrm{L}}\right] d \Omega
$$

is peaked. However, we can see from Eqs. (11) that this distribution in fact oscillates, and it is not peaked at all. Entanglement in noninertial frames can be studied provided we consider the state,

$$
|\Psi\rangle=\frac{1}{\sqrt{2}}\left(\left|0_{\omega}\right\rangle_{\mathrm{M}}\left|0_{\Omega}\right\rangle_{\mathrm{U}}+\left|1_{\omega}\right\rangle_{\mathrm{M}}\left|1_{\Omega}\right\rangle_{\mathrm{U}}\right),
$$

where the states corresponding to $\Omega$ are Unruh states. In this case, a single Unruh frequency $\Omega$ corresponds to the same Rindler frequency. In the special case $q_{\mathrm{R}}=1$ and $q_{\mathrm{L}}=0$, we recover the results canonically presented in the literature $[4,5,22]$. In this section, we will revise the analysis of entanglement in noninertial frames for the general Unruh modes. However, since a Minkowski monochromatic basis seems to be the natural choice for inertial observers, in Sec. IV, we will show that the standard results also hold for Minkowski states, as long as special Minkowski wave packets are considered.

Having the expressions for the vacuum and single-particle states in the Minkowski, Unruh, and Rindler bases enables us to return to the standard scenario for analyzing the degradation of entanglement from the perspective of observers in uniform acceleration. Let us consider the maximally entangled state Eq. (18) from the perspective of inertial observers. By choosing different $q_{\mathrm{R}}$, we can vary the states under consideration. An arbitrary Unruh single-particle state has different right and left components where $q_{\mathrm{R}}, q_{\mathrm{L}}$ represent the respective weights. When working with Unruh modes, there is no particular reason to choose a specific $q_{\mathrm{R}}$. In fact, and as as we will see later, feasible elections of Minkowski states are, in general, linear superpositions of different Unruh modes with different values of $q_{\mathrm{R}}$.

The Minkowski-Unruh state under consideration can be viewed as an entangled state of a tripartite system. The partitions correspond to the three sets of modes: Minkowski modes with frequency $\omega$ and two sets of Unruh modes (left and right) with frequency $\Omega$. Therefore, it is convenient to define the following bipartitions: The Alice-Bob bipartition corresponds to Minkowski and right Unruh modes, while the Alice-antiBob bipartition refers to Minkowski and left Unruh modes. We will see that the distribution of entanglement in these bipartitions becomes relevant when analyzing the entanglement content in the state from the noninertial perspective.

We now want to study the entanglement in the state considering that the $\Omega$ modes are described by observers in uniform acceleration. Therefore, Unruh states must be transformed into the Rindler basis. The state in the MinkowskiRindler basis is also a state of a tripartite system. Therefore, we define the Alice-Rob bipartition as the Minkowski and region I Rindler modes, while the Alice-antiRob bipartitions correspond to Minkowski and region II Rindler modes. In the limit of very small accelerations, Alice-Rob and Alice-antiRob approximate to Alice-Bob and Alice-antiBob bipartitions, respectively. This is because, as shown in Eqs. (7) and (13), region I (II) Rindler modes tend to R (L) Unruh modes in such limits.

The entanglement can be quantified using the Peres partialtranspose criterion. Since the partial transpose of a separable state always has positive eigenvalues, the state is nonseparable (and, therefore, entangled) if the partial-transposed density matrix has, at least, one negative eigenvalue. However, this is a sufficient and necessary condition only for $2 \times 2$ - and $2 \times 3$-dimensional systems. In higher dimensions, the criterion is only necessary. Based on the Peres criterion, a number of entanglement measures has been introduced. In our analysis, we will use the negativity $\mathcal{N}$ to account for the quantum correlations between the different bipartitions of the system. It is defined as the sum of the negative eigenvalues of the partialtranspose density matrix, that is, if $\lambda_{I}$ are the eigenvalues of any partially transposed bipartite density matrix $\rho_{A B}$, then its negativity is

$$
\mathcal{N}_{A B}=\frac{1}{2} \sum_{i}\left(\left|\lambda_{\mathrm{I}}\right|-\lambda_{\mathrm{I}}\right)=-\sum_{\lambda_{\mathrm{I}}<0} \lambda_{\mathrm{I}} .
$$

The maximum value of the negativity (reached for maximally entangled states) depends on the dimension of the maximally entangled state, specifically, for qubits $\mathcal{N}_{A B}^{\max }=1 / 2$.

In what follows, we study the entanglement between the Alice-Rob and Alice-antiRob modes. After expressing Rob's modes in the Rindler basis, the Alice-Rob density matrix is obtained by tracing over region II, with the result,

$$
\rho_{A R}=\frac{1}{2} \sum_{n=0}^{\infty}\left[\frac{\tanh ^{n} r_{\Omega}}{\cosh r_{\Omega}}\right]^{2} \rho_{A R}^{n},
$$

where

$$
\begin{aligned}
\rho_{A R}^{n}= & |0 n\rangle\langle 0 n|+\frac{n+1}{\cosh ^{2} r_{\Omega}}\left(\left|q_{\mathrm{R}}\right|^{2}|1 n+1\rangle\langle 1 n+1|\right. \\
& \left.+\left|q_{\mathrm{L}}\right|^{2}|1 n\rangle\langle 1 n|\right)+\frac{\sqrt{n+1}}{\cosh r_{\Omega}}\left(q_{\mathrm{R}}|1 n+1\rangle\langle 0 n|\right. \\
& \left.+q_{\mathrm{L}} \tanh r_{\Omega}|1 n\rangle\langle 0 n+1|\right)+\frac{\sqrt{(n+1)(n+2)}}{\cosh ^{2} r_{\Omega}} \\
& \times q_{\mathrm{R}} q_{\mathrm{L}}^{*} \tanh r_{\Omega}|1 n+2\rangle\langle 1 n|+(\text { H.c. })_{\text {nondiag. }} .
\end{aligned}
$$

Here, (H.c.) nondiag means Hermitian conjugate of only the nondiagonal terms. The Alice-antiRob density matrix is obtained by tracing over region I. However, due to the symmetry in the Unruh modes between regions I and II, we can obtain the Alice-antiRob matrix by exchanging $q_{\mathrm{R}}$ and $q_{\mathrm{L}}$. The partial transpose $\sigma_{\mathrm{R}}$ of $\rho_{\mathrm{R}}$ with respect to Alice is given by

$$
\sigma_{A R}=\frac{1}{2} \sum_{n=0}^{\infty}[f(n)]^{2} \sigma_{A R}^{n},
$$

where

$$
\begin{aligned}
\sigma_{A R}^{n}= & |0 n\rangle\langle 0 n|+\frac{n+1}{\cosh ^{2} r_{\Omega}}\left(\left|q_{\mathrm{R}}\right|^{2}|1 n+1\rangle\langle 1 n+1|\right. \\
& \left.+\left|q_{\mathrm{L}}\right|^{2}|1 n\rangle\langle 1 n|\right)+\frac{\sqrt{n+1}}{\cosh r_{\Omega}}\left(q_{\mathrm{R}}|0 n+1\rangle\langle 1 n|\right. \\
& \left.+q_{\mathrm{L}} \tanh r_{\Omega}|0 n\rangle\langle 1 n+1|\right)+\frac{\sqrt{(n+1)(n+2)}}{\cosh ^{2} r_{\Omega}} \\
& \times q_{\mathrm{R}} q_{\mathrm{L}}^{*} \tanh r_{\Omega}|1 n+2\rangle\langle 1 n|+(\text { H.c. })_{\text {nondiag. }} .
\end{aligned}
$$




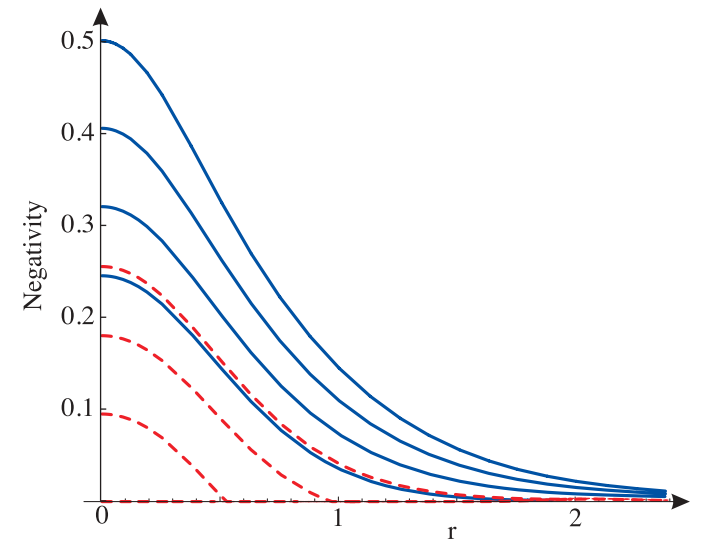

FIG. 2. (Color online) Negativity for the bipartition Alice-Rob (blue continuous) and Alice-antiRob (red dashed) as a function of $r_{\Omega}=\arctan e^{-\pi \Omega_{a} / a}$ for various choices of $\left|q_{\mathrm{R}}\right|$. The blue continuous (red dashed) curves from top to bottom (from bottom to top) correspond to $\left|q_{\mathrm{R}}\right|=1,0.9,0.8,0.7$, respectively.

The eigenvalues of $\sigma_{A R}$ only depend on $\left|q_{\mathrm{R}}\right|$ and $\left|q_{\mathrm{L}}\right|$ and not on the relative phase between them. This means that the entanglement is insensitive to the election of this phase.

The two extreme cases when $q_{R}=1$ and $q_{L}=1$ are analytically solvable, since the partial-transpose density matrix has a block-diagonal structure as shown in previous works [4]. However, for all other cases, the matrix is no longer block diagonal, and the eigenvalues of the partial-transpose density matrix are computed numerically. The resulting negativity between Alice-Rob and Alice-antiRob modes is plotted in Fig. 2 for different values of $\left|q_{\mathrm{R}}\right|=1,0.9,0.8,0.7$. $\left|q_{\mathrm{R}}\right|=1$ corresponds to the canonical case studied in the literature [4].

In the bosonic case, the entanglement between the AliceRob and the Alice-antiRob modes always vanishes in the infinite-acceleration limit. Interestingly, there is no fundamental difference in the degradation of entanglement for different choices of $\left|q_{\mathrm{R}}\right|$. The entanglement always degrades with acceleration at the same rate. There is no special Unruh state, which degrades less with acceleration.

\section{WAVE PACKETS: RECOVERING THE SINGLE-MODE APPROXIMATION}

The entanglement analysis of Sec. III assumes Alice's state to be a Minkowski particle with a sharp Minkowski momentum and Rob's state to be an Unruh particle with sharp Unruh frequency, such that Rob's linear combination of the two Unruh modes is specified by the two complex-valued parameters $q_{\mathrm{R}}$ and $q_{\mathrm{L}}$ satisfying $\left|q_{\mathrm{R}}\right|^{2}+\left|q_{\mathrm{L}}\right|^{2}=1$. The Alice and Rob states are further assumed to be orthogonal to each other so that the system can be treated as bipartite. We now discuss the sense in which these assumptions are a good approximation to Alice and Rob states that can be built as Minkowski wave packets.

Recall that a state with a sharp frequency, be it Minkowski or Unruh, is not normalizable and should be understood as the idealization of a wave packet that contains a continuum of frequencies with an appropriate peaking. Suppose that the Alice and Rob states are initially set up as Minkowski wave packets, peaked about distinct Minkowski momenta and having negligible overlap so that the bipartite assumption is a good approximation. The transformation between the Minkowski and the Unruh bases is an integral transform, given by Eqs. (9) and (11): Can the Rob state be arranged to be peaked about a single Unruh frequency? If so, how are the frequency uncertainties on the Minkowski and Unruh sides related?

\section{A. Massless scalar field}

We focus first on the massless scalar field of Sec. III. The massive scalar field will be discussed in Sec. IV B. We expect the analysis for fermions to be qualitatively similar.

Consider a packet of Minkowski creation operators $a_{\omega, \mathrm{M}}^{\dagger}$ smeared with a weight function $f(\omega)$. We wish to express this packet in terms of Unruh creation operators $A_{\Omega, \mathrm{R}}^{\dagger}$ and $A_{\Omega, \mathrm{L}}^{\dagger}$ smeared with the weight functions $g_{\mathrm{R}}(\Omega)$ and $g_{\mathrm{L}}(\Omega)$ so that

$$
\int_{0}^{\infty} f(\omega) a_{\omega, \mathrm{M}}^{\dagger} d \omega=\int_{0}^{\infty}\left[g_{\mathrm{R}}(\Omega) A_{\Omega, \mathrm{R}}^{\dagger}+g_{\mathrm{L}}(\Omega) A_{\Omega, \mathrm{L}}^{\dagger}\right] d \Omega .
$$

From Eq. (12), it follows that the smearing functions are related by

$$
\begin{gathered}
g_{\mathrm{R}}(\Omega)=\int_{0}^{\infty} \alpha_{\omega \Omega}^{\mathrm{R}} f(\omega) d \omega, \\
g_{\mathrm{R}}(\Omega)=\int_{0}^{\infty} \alpha_{\omega \Omega}^{\mathrm{R}} f(\omega) d \omega, \\
f(\omega)=\int_{0}^{\infty}\left[\left(\alpha_{\omega \Omega}^{\mathrm{R}}\right)^{*} g_{\mathrm{R}}(\Omega)+\left(\alpha_{\omega \Omega}^{\mathrm{L}}\right)^{*} g_{\mathrm{L}}(\Omega)\right] d \Omega .
\end{gathered}
$$

By Eq. (11), Eqs. (25) are recognized as a Fourier transform pair between the variable $\ln (\omega l) \in \mathbb{R}$ on the Minkowski side and the variable $\pm \Omega \in \mathbb{R}$ on the Unruh side: The full real line on the Unruh side has been broken into the Unruh frequency $\Omega \in \mathbb{R}^{+}$and the discrete index $\mathrm{R}$, L. Thus, all standard properties of Fourier transforms apply. Parseval's theorem takes the form

$$
\int_{0}^{\infty}|f(\omega)|^{2} d \omega=\int_{0}^{\infty}\left[\left|g_{\mathrm{R}}(\Omega)\right|^{2}+\left|g_{\mathrm{L}}(\Omega)\right|^{2}\right] d \Omega,
$$

and the uncertainty relation reads

$$
(\Delta \Omega)[\Delta \ln (\omega l)] \geqslant \frac{1}{2},
$$

where $\Delta \Omega$ is understood by combining contributions from $g_{\mathrm{R}}(\Omega)$ and $g_{\mathrm{L}}(\Omega)$ in the sense of Eq. (25). Note that, since equality in Eq. (27) holds only for Gaussians, any state in which one of $g_{\mathrm{R}}(\Omega)$ and $g_{\mathrm{L}}(\Omega)$ vanishes will satisfy Eq. (27) with a genuine inequality.

As a concrete example, with a view to optimizing the peaking both in Minkowski frequency and in Unruh frequency, consider a Minkowski smearing function that is a Gaussian in $\ln (\omega l)$,

$$
f(\omega)=\left(\frac{\lambda}{\pi \omega^{2}}\right)^{1 / 4} \exp \left\{-\frac{1}{2} \lambda\left[\ln \left(\omega / \omega_{0}\right)\right]^{2}\right\}\left(\omega / \omega_{0}\right)^{-i \mu},
$$

where $\omega_{0}$ and $\lambda$ are positive parameters and $\mu$ is a realvalued parameter. $\lambda$ and $\mu$ are dimensionless, and $\omega_{0}$ has the dimension of inverse length. Note that $f$ is normalized, 
$\int_{0}^{\infty}|f(\omega)|^{2} d \omega=1$. The expectation value and uncertainty of $\ln (\omega l)$ are those of a standard Gaussian, $\langle\ln (\omega l)\rangle=\ln \left(\omega_{0} l\right)$ and $\Delta \ln (\omega l)=(2 \lambda)^{-1 / 2}$, while the expectation value and uncertainty of $\omega$ are given by

$$
\begin{gathered}
\langle\omega\rangle=\exp \left(\frac{1}{4} \lambda^{-1}\right), \\
\Delta \omega=\langle\omega\rangle\left[\exp \left(\frac{1}{2} \lambda^{-1}\right)-1\right]^{1 / 2} .
\end{gathered}
$$

The Unruh smearing functions are cropped Gaussians,

$$
\begin{gathered}
g_{\mathrm{R}}(\Omega)=\frac{1}{(\pi \lambda)^{1 / 4}} \exp \left[-\frac{1}{2} \lambda^{-1}(\Omega-\epsilon \mu)^{2}\right]\left(\omega_{0} l\right)^{i \epsilon \Omega}, \\
g_{\mathrm{L}}(\Omega)=\frac{1}{(\pi \lambda)^{1 / 4}} \exp \left[-\frac{1}{2} \lambda^{-1}(\Omega+\epsilon \mu)^{2}\right]\left(\omega_{0} l\right)^{-i \epsilon \Omega} .
\end{gathered}
$$

For $\epsilon \mu \gg \lambda^{1 / 2}, g_{\mathrm{L}}(\Omega)$ is small and $g_{\mathrm{R}}(\Omega)$ is peaked around $\Omega=\epsilon \mu$ with uncertainty $(\lambda / 2)^{1 / 2}$; conversely, for $\epsilon \mu \ll$ $-\lambda^{1 / 2}, g_{\mathrm{R}}(\Omega)$ is small and $g_{\mathrm{L}}(\Omega)$ is peaked around $\Omega=$ $-\epsilon \mu$ with uncertainty $(\lambda / 2)^{1 / 2}$. Note that, in these limits, the relative magnitudes of $g_{\mathrm{L}}(\Omega)$ and $g_{\mathrm{R}}(\Omega)$ are consistent with the magnitude of the smeared mode Minkowski mode function $\int_{0}^{\infty} f(\omega) u_{\omega, \mathrm{M}} d \omega$ in the corresponding regions of Minkowski space: A contour deformation argument shows that, for $\epsilon \mu \gg \lambda^{1 / 2}$, the smeared mode function is large in the region $t+x>0$ and small in the region $t+x<0$, while for $\epsilon \mu \ll-\lambda^{1 / 2}$, it is large in the region $t-x>0$ and small in the region $t-x<0$.

Now, let the Rob state have the smearing function (28), and choose for Alice any state that has negligible overlap with the Rob state, for example, by taking for Alice and Rob distinct values of $\epsilon$. For $|\mu| \gg \lambda^{1 / 2}$ and $\lambda$ not larger than of order unity, the combined state is then well approximated by the single Unruh frequency state of Sec. III with $\Omega=|\mu|$ and with one of $q_{\mathrm{R}}$ and $q_{\mathrm{L}}$ vanishing. Hence, in this case, we recover the results in Ref. [4]. To build a Rob state that is peaked about a single Unruh frequency with comparable $q_{\mathrm{R}}$ and $q_{\mathrm{L}}$ so that the results of Sec. III are recovered, we may take a Minkowski smearing function that is a linear combination of Eq. (28) and its complex conjugate.

While the phase factor $\left(\omega / \omega_{0}\right)^{-i \mu}$ in the Minkowski smearing function (28) is essential for adjusting the locus of the peak in the Unruh smearing functions, the choice of a logarithmic Gaussian for the magnitude appears nonessential. We have verified that similar results ensue with the choices

$$
f(\omega)=\frac{2^{\lambda}\left(\omega / \omega_{0}\right)^{\lambda-i \mu} \exp \left(-\omega / \omega_{0}\right)}{\sqrt{\omega \Gamma(2 \lambda)}},
$$

and

$$
f(\omega)=\frac{\left(\omega / \omega_{0}\right)^{-i \mu}}{\sqrt{2 \omega K_{0}(2 \lambda)}} \exp \left[-\frac{\lambda}{2}\left(\frac{\omega}{\omega_{0}}+\frac{\omega_{0}}{\omega}\right)\right],
$$

for which the respective Unruh smearing functions can be expressed, respectively, in terms of the $\gamma$ function and a modified Bessel function.

\section{B. Massive scalar field}

For a scalar field of mass $m>0$, the Minkowski modes of the Klein-Gordon equation are

$$
u_{k, \mathrm{M}}(t, x)=\frac{1}{\sqrt{4 \pi \omega}} \exp (-i \omega t+i k x),
$$

where $k \in \mathbb{R}$ is the Minkowski momentum and $\omega \equiv \omega_{k}=$ $\sqrt{m^{2}+k^{2}}$ is the Minkowski frequency. These modes are $\delta$ normalized in $k$ as usual. The Rindler modes are [37]

$$
\begin{gathered}
u_{\Omega, \mathrm{I}}(t, x)=N_{\Omega} \exp \left[-\frac{i \Omega}{2} \ln \left(\frac{x+t}{x-t}\right)\right], \\
u_{\Omega, \mathrm{II}}(t, x)=N_{\Omega} \exp \left[-\frac{i \Omega}{2} \ln \left(\frac{-x+t}{-x-t}\right)\right],
\end{gathered}
$$

where $N_{\Omega}=\frac{\sqrt{\sinh \pi \Omega}}{\pi} K_{i \Omega}\left(m \sqrt{x^{2}-t^{2}}\right)$ and $\Omega>0$ is the (dimensionless) Rindler frequency. These modes are $\delta$ normalized in $\Omega$. The Unruh modes $u_{\Omega, \mathrm{R}}$ and $u_{\Omega, \mathrm{L}}$ are as in Eq. (7). Note that, in the Minkowski modes (33), the distinction between the left movers and the right movers is in the sign of the label $k \in \mathbb{R}$, but, in the Rindler and Unruh modes, the right movers and the left movers do not decouple, owing to the asymptotic behavior of the solutions at the Rindler spatial infinity. Therefore, the Rindler and Unruh modes do not carry an index $\epsilon$ that would distinguish the right movers and the left movers.

The transformation between the Minkowski and the Unruh modes can be found by the methods of Ref. [37]. In our notation, the transformation reads

$$
\begin{gathered}
u_{\Omega, \mathrm{R}}=\int_{-\infty}^{\infty}\left(\alpha_{k \Omega}^{\mathrm{R}}\right)^{*} u_{k, \mathrm{M}} d k, \\
u_{\Omega, \mathrm{L}}=\int_{-\infty}^{\infty}\left(\alpha_{k \Omega}^{\mathrm{L}}\right)^{*} u_{k, \mathrm{M}} d k, \\
u_{k, \mathrm{M}}=\int_{0}^{\infty}\left(\alpha_{k \Omega}^{\mathrm{R}} u_{\Omega, \mathrm{R}}+\alpha_{k \Omega}^{\mathrm{L}} u_{\Omega, \mathrm{L}}\right) d \Omega,
\end{gathered}
$$

where

$$
\begin{aligned}
\alpha_{k \Omega}^{\mathrm{R}} & =\frac{1}{\sqrt{2 \pi \omega}}\left(\frac{\omega+k}{m}\right)^{i \Omega}, \\
\alpha_{k \Omega}^{\mathrm{L}} & =\frac{1}{\sqrt{2 \pi \omega}}\left(\frac{\omega+k}{m}\right)^{-i \Omega} .
\end{aligned}
$$

Hence, transformations for the various operators read as in Sec. III but with the replacements,

$$
\omega \rightarrow k, \quad \int_{0}^{\infty} d \omega \longrightarrow \int_{-\infty}^{\infty} d k
$$

and no $\epsilon$. In particular,

$$
\begin{gathered}
a_{\Omega, \mathrm{R}}=\int_{-\infty}^{\infty} \alpha_{k \Omega}^{\mathrm{R}} a_{k, \mathrm{M}} d k, \\
a_{\Omega, \mathrm{L}}=\int_{-\infty}^{\infty} \alpha_{k \Omega}^{\mathrm{L}} a_{k, \mathrm{M}} d k, \\
a_{k, \mathrm{M}}=\int_{0}^{\infty}\left[\left(\alpha_{k \Omega}^{\mathrm{R}}\right)^{*} A_{\Omega, \mathrm{R}}+\left(\alpha_{k \Omega}^{\mathrm{L}}\right)^{*} A_{\Omega, \mathrm{L}}\right] d \Omega .
\end{gathered}
$$

To consider peaking of Minkowski wave packets in the Unruh frequency, we note that the transform (38) with Eq. (36) 
is now a Fourier transform between the Minkowski rapidity $\tanh ^{-1}(k / \omega)=\ln [(\omega+k) / m] \in \mathbb{R}$ and $\pm \Omega \in \mathbb{R}$. Hence, the bulk of the massless peaking discussion of Sec. IV A goes through with the replacements (37) and $\omega l \rightarrow(\omega+k) / m$. The main qualitative difference is that, in the massive case, one cannot appeal to the decoupling of the right movers and left movers when choosing for Alice and Rob states that have negligible overlap.

\section{UNRUH ENTANGLEMENT DEGRADATION FOR DIRAC FIELDS}

Statistics plays a very important role in the behavior of entanglement described by observers in uniform acceleration. While entanglement vanishes in the limit of infinite acceleration in the bosonic case [4,22], it remains finite for Dirac fields $[5,15]$. Therefore, it is interesting to revise the analysis of entanglement between Dirac fields for different elections of Unruh modes.

\section{A. Dirac fields}

In a parallel analysis to the bosonic case, we consider a Dirac field $\phi$ satisfying the equation $\left\{i \gamma^{\mu}\left(\partial_{\mu}-\Gamma_{\mu}\right)+m\right\} \phi=$ 0 where $\gamma^{\mu}$ are the Dirac-Pauli matrices and $\Gamma_{\mu}$ are spinorial affine connections. The field expansion in terms of the Minkowski solutions of the Dirac equation is

$$
\phi=N_{\mathrm{M}} \sum_{k}\left(c_{k, \mathrm{M}} u_{k, \mathrm{M}}^{+}+d_{k, \mathrm{M}}^{\dagger} u_{k, \mathrm{M}}^{-}\right),
$$

where $N_{\mathrm{M}}$ is a normalization constant and the label \pm denotes positive and negative energy solutions, respectively (particles and antiparticles) with respect to the Minkowskian Killing vector field $\partial_{t}$. The label $k$ is a multilabel including energy and spin $k=\left\{E_{\omega}, s\right\}$ where $s$ is the component of the spin on the quantization direction. $c_{k}$ and $d_{k}$ are the particle and antiparticle operators that satisfy the usual anticommutation rule,

$$
\left\{c_{k, \mathrm{M}}, c_{k^{\prime}, \mathrm{M}}^{\dagger}\right\}=\left\{d_{k, \mathrm{M}}, d_{k^{\prime}, \mathrm{M}}^{\dagger}\right\}=\delta_{k k^{\prime}},
$$

and all other anticommutators vanishing. The Dirac-field operator, in terms of Rindler modes, is given by

$$
\phi=N_{\mathrm{R}} \sum_{j}\left(c_{j, \mathrm{I}} u_{j, \mathrm{I}}^{+}+d_{j, \mathrm{I}}^{\dagger} u_{j, \mathrm{I}}^{-}+c_{j, \mathrm{II}} u_{j, \mathrm{II}}^{+}+d_{j, \mathrm{II}}^{\dagger} u_{j, \mathrm{II}}^{-}\right),
$$

where $N_{\mathrm{R}}$ is, again, a normalization constant. $c_{j, \Sigma}, d_{j, \Sigma}$ with $\Sigma=$ I, II represent Rindler particle and antiparticle operators. The usual anticommutation rules again apply. Note that operators in different regions $\Sigma=$ I, II do not commute but anticommute. $j=\left\{E_{\Omega}, s^{\prime}\right\}$ is again a multilabel including all the degrees of freedom. Here, $u_{k, \mathrm{I}}^{ \pm}$and $u_{k, \mathrm{II}}^{ \pm}$are the positive- and negative-frequency solutions of the Dirac equation in Rindler coordinates with respect to the Rindler timelike Killing vector field in regions I and II, respectively. The modes $u_{k, \mathrm{I}}^{ \pm}, u_{k, \mathrm{II}}^{ \pm}$do not have support outside the right, left Rindler wedges. The annihilation operators $c_{k, \mathrm{M}}, d_{k, \mathrm{M}}$ define the Minkowski vacuum $|0\rangle_{\mathrm{M}}$, which must satisfy

$$
c_{k, \mathrm{M}}|0\rangle_{\mathrm{M}}=d_{k, \mathrm{M}}|0\rangle_{\mathrm{M}}=0, \quad \forall k .
$$

In the same fashion, $c_{j, \Sigma}, d_{j, \Sigma}$ define the Rindler vacua in regions $\Sigma=\mathrm{I}$,II,

$$
c_{j, \mathrm{R}}|0\rangle_{\Sigma}=d_{j, \mathrm{R}}|0\rangle_{\Sigma}=0, \quad \forall j, \Sigma=\mathrm{I}, \mathrm{II} .
$$

The transformation between the Minkowski and the Rindler modes is given by

$$
u_{j, \mathrm{M}}^{+}=\sum_{k}\left[\alpha_{j k}^{\mathrm{I}} u_{k, \mathrm{I}}^{+}+\beta_{j k}^{\mathrm{I} *} u_{k, \mathrm{I}}^{-}+\alpha_{j k}^{\mathrm{II}} u_{k, \mathrm{II}}^{+}+\beta_{j k}^{\mathrm{II} *} u_{k, \mathrm{II}}^{-}\right] .
$$

The coefficients that relate both sets of modes are given by the inner product,

$$
\left(u_{k}, u_{j}\right)=\int d^{3} x u_{k}^{\dagger} u_{j},
$$

so that the Bogoliubov coefficients are, after some elementary but lengthy algebra [41,42],

$$
\begin{gathered}
\alpha_{j k}^{\mathrm{I}}=e^{i \theta E_{\Omega}} \frac{1+i}{2 \sqrt{\pi E_{\omega}}} \frac{e^{\pi E_{\Omega} / 2}}{\sqrt{e^{\pi E_{\Omega}}+e^{-\pi E_{\Omega}}}} \delta_{s s^{\prime}}, \\
\beta_{j k}^{\mathrm{I}}=-e^{i \theta E_{\Omega}} \frac{1+i}{2 \sqrt{\pi E_{\omega}}} \frac{e^{-\pi E_{\Omega} / 2}}{\sqrt{e^{\pi E_{\Omega}}+e^{-\pi E_{\Omega}}}} \delta_{s s^{\prime}},
\end{gathered}
$$

where $E_{\Omega}$ is the energy of the Rindler mode $k, E_{\omega}$ is the energy of the Minkowski mode $j$, and $\theta$ is a parameter defined such that it satisfies the condition $E_{\Omega}=m \cosh \theta$ and $\left|\boldsymbol{k}_{\Omega}\right|=$ $m \sinh \theta$ (see Ref. [41]). One can verify that $\alpha^{\mathrm{II}}=\left(\alpha^{\mathrm{I}}\right)^{*}$ and $\beta^{\mathrm{II}}=\left(\beta^{\mathrm{I}}\right)^{*}$. Defining $\tan r_{\Omega}=e^{-\pi E_{\Omega}}$, the coefficients become

$$
\begin{gathered}
\alpha_{j k}^{\mathrm{I}}=e^{i \theta E_{\Omega}} \frac{1+i}{2 \sqrt{\pi E_{\omega}}} \cos r_{\Omega} \delta_{s s^{\prime}}, \\
\beta_{j k}^{\mathrm{I}}=-e^{i \theta E_{\Omega}} \frac{1+i}{2 \sqrt{\pi E_{\omega}}} \sin r_{\Omega} \delta_{s s^{\prime}} .
\end{gathered}
$$

Finally, taking into account that $c_{j, \mathrm{M}}=\left(u_{j, \mathrm{M}}^{+}, \phi\right)$, we find the Minkowski particle annihilation operator to be

$$
c_{j, \mathrm{M}}=\sum_{k}\left[\alpha_{j k}^{\mathrm{I} *} c_{k, \mathrm{I}}+\beta_{j k}^{\mathrm{I}} d_{k, s, \mathrm{I}}^{\dagger}+\alpha_{j k}^{\mathrm{II} *} c_{k, \mathrm{II}}+\beta_{j k}^{\mathrm{II}} d_{k, \mathrm{II}}^{\dagger}\right] \text {. }
$$

We now consider the transformations between states in different bases. For this, we define an arbitrary element of the Dirac-field Fock basis for each mode as

$$
\left|F_{k}\right\rangle=\left|F_{k}\right\rangle_{\mathrm{R}} \otimes\left|F_{k}\right\rangle_{\mathrm{L}},
$$

where

$$
\begin{aligned}
\left|F_{k}\right\rangle_{\mathrm{R}} & =|n\rangle_{\mathrm{I}}^{+}|m\rangle_{\mathrm{II}}^{-}, \\
\left|F_{k}\right\rangle_{\mathrm{L}} & =|p\rangle_{\mathrm{I}}^{-}|q\rangle_{\mathrm{II}}^{+} .
\end{aligned}
$$

Here, the \pm indicates particle and antiparticle. Operating with the Bogoliubov coefficients, making this tensor product structure explicit, we obtain

$$
c_{j, \mathrm{M}}=N_{j} \sum_{k}\left[\chi^{*}\left(C_{k, \mathrm{R}} \otimes \mathbb{1}_{\mathrm{L}}\right)+\chi\left(\mathbb{1}_{\mathrm{R}} \otimes C_{k, \mathrm{~L}}\right)\right],
$$

where

$$
N_{j}=\frac{1}{2 \sqrt{\pi E_{\omega}}}, \quad \chi=(1+i) e^{i \theta E_{\Omega}},
$$


and the operators,

$$
\begin{aligned}
C_{k, \mathrm{R}} & \equiv\left(\cos r_{k} c_{k, \mathrm{I}}-\sin r_{k} d_{k, \mathrm{II}}^{\dagger}\right), \\
C_{k, \mathrm{~L}} & \equiv\left(\cos r_{k} c_{k, \mathrm{II}}-\sin r_{k} d_{k, \mathrm{I}}^{\dagger}\right)
\end{aligned}
$$

are the so-called Unruh operators.

It can be shown [43] that, for a massless Dirac field, the Unruh operators have the same form as Eq. (54), however, in this case, $\tan r_{k}=e^{-\pi \Omega_{a} / a}$.

In the massless case, to find the Minkowski vacuum in the Rindler basis, we consider the following ansatz,

$$
|0\rangle_{\mathrm{M}}=\bigotimes_{\Omega}\left|0_{\Omega}\right\rangle_{\mathrm{M}}
$$

where $\left|0_{\Omega}\right\rangle_{\mathrm{M}}=\left|0_{\Omega}\right\rangle_{\mathrm{R}} \otimes\left|0_{\Omega}\right\rangle_{\mathrm{L}}$. We find that

$$
\begin{aligned}
\left|0_{\Omega}\right\rangle_{\mathrm{R}} & =\sum_{n, s}\left(F_{n, \Omega, s}\left|n_{\Omega, s}\right\rangle_{\mathrm{I}}^{+}\left|n_{\Omega,-s}\right\rangle_{\mathrm{II}}^{-}\right), \\
\left|0_{\Omega}\right\rangle_{\mathrm{L}} & =\sum_{n, s}\left(G_{n, \Omega, s}\left|n_{\Omega, s}\right\rangle_{\mathrm{I}}^{-}\left|n_{\Omega,-s}\right\rangle_{\mathrm{II}}^{+}\right),
\end{aligned}
$$

where the label \pm denotes particle and antiparticle modes and $s$ labels the spin. The minus signs on the spin label in region II show explicitly that spin, as all the magnitudes, which change under time reversal, is opposite in region I with respect to region II.

We obtain the form of the coefficients $F_{n, \Omega, s}, G_{n, \Omega, s}$ for the vacuum by imposing that the Minkowski vacuum is annihilated by the particle annihilator for all frequencies and values for the spin third component.

\section{B. Grassman scalars}

Since the simplest case that preserves the fundamental Dirac characteristics corresponds to Grassman scalars, we study them in what follows. Moreover, the entanglement in noninertial frames between scalar fermionic fields has been extensively studied under the single-mode approximation in the literature [5]. In this case, the Pauli exclusion principle limits the sums (56), and only the two following terms contribute

$$
\begin{aligned}
& \left|0_{\Omega}\right\rangle_{\mathrm{R}}=F_{0}\left|0_{\Omega}\right\rangle_{\mathrm{I}}^{+}\left|0_{\Omega}\right\rangle_{\mathrm{II}}^{-}+F_{1}\left|1_{\Omega}\right\rangle_{\mathrm{I}}^{+}\left|1_{\Omega}\right\rangle_{\mathrm{II}}^{-}, \\
& \left|0_{\Omega}\right\rangle_{\mathrm{L}}=G_{0}\left|0_{\Omega}\right\rangle_{\mathrm{I}}^{-}\left|0_{\Omega}\right\rangle_{\mathrm{II}}^{+}+G_{1}\left|1_{\Omega}\right\rangle_{\mathrm{I}}^{-}\left|1_{\Omega}\right\rangle_{\mathrm{II}}^{+} .
\end{aligned}
$$

Due to the anticommutation relations, we must introduce the following sign conventions,

$$
\begin{aligned}
\left|1_{\Omega}\right\rangle_{\mathrm{I}}^{+}\left|1_{\Omega}\right\rangle_{\mathrm{II}}^{-} & =d_{\Omega, \mathrm{II}}^{\dagger} c_{\Omega, \mathrm{I}}^{\dagger}\left|0_{\Omega}\right\rangle_{\mathrm{I}}^{+}\left|0_{\Omega}\right\rangle_{\mathrm{II}}^{-} \\
& =-c_{\Omega, \mathrm{I}}^{\dagger} d_{\Omega, \mathrm{II}}^{\dagger}\left|0_{\Omega}\right\rangle_{\mathrm{I}}^{+}\left|0_{\Omega}\right\rangle_{\mathrm{II}}^{-}, \\
\left|1_{\Omega}\right\rangle_{\mathrm{I}}^{-}\left|1_{\Omega}\right\rangle_{\mathrm{II}}^{+} & =c_{\Omega, \mathrm{II}}^{\dagger} d_{\Omega, \mathrm{I}}^{\dagger}\left|0_{\Omega}\right\rangle_{\mathrm{I}}^{-}\left|0_{\Omega}\right\rangle_{\mathrm{II}}^{+} \\
& =-d_{\Omega, \mathrm{I}}^{\dagger} c_{\Omega, \mathrm{II}}^{\dagger}\left|0_{\Omega}\right\rangle_{\mathrm{I}}^{-}\left|0_{\Omega}\right\rangle_{\mathrm{II}}^{+} .
\end{aligned}
$$

We obtain the form of the coefficients by imposing that $c_{\omega, \mathrm{M}}\left|0_{\Omega}\right\rangle_{\mathrm{M}}=0$, which translates into $C_{\Omega, \mathrm{R}}\left|0_{\Omega}\right\rangle_{\mathrm{R}}=$ $C_{\Omega, \mathrm{L}}\left|0_{\Omega}\right\rangle_{\mathrm{L}}=0$. Therefore,

$$
\begin{aligned}
C_{\Omega, \mathrm{R}}\left(F_{0}\left|0_{\Omega}\right\rangle_{\mathrm{I}}^{+}\left|0_{\Omega}\right\rangle_{\mathrm{II}}^{-}+F_{1}\left|1_{\Omega}\right\rangle_{\mathrm{I}}^{+}\left|1_{\Omega}\right\rangle_{\mathrm{II}}^{-}\right) & =0, \\
C_{\Omega, \mathrm{L}}\left(G_{0}\left|0_{\Omega}\right\rangle_{\mathrm{I}}^{-}\left|0_{\Omega}\right\rangle_{\mathrm{II}}^{+}+G_{1}\left|1_{\Omega}\right\rangle_{\mathrm{I}}^{-}\left|1_{\Omega}\right\rangle_{\mathrm{II}}^{+}\right) & =0 .
\end{aligned}
$$

These conditions imply that

$$
\begin{array}{ccc}
F_{1} \cos r_{\Omega}-F_{0} \sin r_{\Omega}=0 & \Rightarrow & F_{1}=F_{0} \tan r_{\Omega}, \\
G_{1} \cos r_{\Omega}+G_{0} \sin r_{\Omega}=0 & \Rightarrow & G_{1}=-G_{0} \tan r_{\Omega},
\end{array}
$$

which, together with the normalization conditions $\left\langle\left. 0_{\Omega}\right|_{R} \| 0_{\Omega}\right\rangle_{R}=1$ and $\left\langle\left. 0_{\Omega}\right|_{L} \mid 0_{\Omega}\right\rangle_{L}=1$, yield

$$
\begin{aligned}
F_{0} & =\cos r_{\Omega}, \quad F_{1}=\sin r_{\Omega}, \\
G_{0} & =\cos r_{\Omega} \quad G_{1}=-\sin r_{\Omega} .
\end{aligned}
$$

Therefore, the vacuum state is given by

$$
\begin{aligned}
\left|0_{\Omega}\right\rangle= & \left(\cos r_{\Omega}\left|0_{\Omega}\right\rangle_{\mathrm{I}}^{+}\left|0_{\Omega}\right\rangle_{\mathrm{II}}^{-}+\sin r_{\Omega}\left|1_{\Omega}\right\rangle_{\mathrm{I}}^{+}\left|1_{\Omega}\right\rangle_{\mathrm{II}}^{-}\right) \\
& \otimes\left(\cos r_{\Omega}\left|0_{\Omega}\right\rangle_{\mathrm{I}}^{-}\left|0_{\Omega}\right\rangle_{\mathrm{II}}^{+}-\sin r_{\Omega}\left|1_{\Omega}\right\rangle_{\mathrm{I}}^{-}\left|1_{\Omega}\right\rangle_{\mathrm{II}}^{+}\right),
\end{aligned}
$$

which is compatible with the result obtained with the Unruh modes. For convenience, we introduce the following notation:

$$
\left|n n^{\prime} n^{\prime \prime} n^{\prime \prime \prime}\right\rangle_{\Omega} \equiv\left|n_{\Omega}\right\rangle_{\mathrm{I}}^{+}\left|n_{\Omega}^{\prime}\right\rangle_{\mathrm{II}}^{-}\left|n_{\Omega}^{\prime \prime}\right\rangle_{\mathrm{I}}^{-}\left|n_{\Omega}^{\prime \prime \prime}\right\rangle_{\mathrm{II}}^{+},
$$

in which the vacuum state is written as

$$
\begin{aligned}
\left|0_{\Omega}\right\rangle= & \cos ^{2} r_{\Omega}|0000\rangle_{\Omega}-\sin r_{\Omega} \cos r_{\Omega}|0011\rangle_{\Omega} \\
& +\sin r_{\Omega} \cos r_{\Omega}|1100\rangle_{\Omega}-\sin ^{2} r_{\Omega}|1111\rangle_{\Omega} .
\end{aligned}
$$

The Minkowskian one-particle state is obtained by applying the creation operator to the vacuum state $\left|1_{j}\right\rangle_{\mathrm{U}}=c_{\Omega, \mathrm{U}}^{\dagger}|0\rangle_{\mathrm{M}}$, where the Unruh particle creator is a combination of the two Unruh operators $C_{\Omega, \mathrm{R}}^{\dagger}$ and $C_{\Omega, \mathrm{L}}^{\dagger}$,

$$
c_{k, \mathrm{U}}^{\dagger}=q_{\mathrm{R}}\left(C_{\Omega, \mathrm{R}}^{\dagger} \otimes \mathbb{1}_{\mathrm{L}}\right)+q_{\mathrm{L}}\left(\mathbb{1}_{\mathrm{R}} \otimes C_{\Omega, \mathrm{L}}^{\dagger}\right) .
$$

Since

$$
\begin{aligned}
& C_{\Omega, \mathrm{R}}^{\dagger} \equiv\left(\cos r_{\Omega} c_{\Omega, \mathrm{I}}^{\dagger}-\sin r_{\Omega} d_{\Omega, \mathrm{II}}\right), \\
& C_{\Omega, \mathrm{L}}^{\dagger} \equiv\left(\cos r_{\Omega} c_{\Omega, \mathrm{II}}^{\dagger}-\sin r_{\Omega} d_{\Omega, \mathrm{I}}\right),
\end{aligned}
$$

with $q_{\mathrm{R}}, q_{\mathrm{L}}$ complex numbers satisfying $\left|q_{\mathrm{R}}\right|^{2}+\left|q_{\mathrm{L}}\right|^{2}=1$, we obtain

$$
\begin{aligned}
& \left|1_{\Omega}\right\rangle_{\mathrm{R}}^{+}=C_{\Omega, \mathrm{R}}^{\dagger}\left|0_{\Omega}\right\rangle_{\mathrm{R}}=\left|1_{\Omega}\right\rangle_{\mathrm{I}}^{+}\left|0_{\Omega}\right\rangle_{\mathrm{II}}^{-}, \\
& \left|1_{\Omega}\right\rangle_{\mathrm{L}}^{+}=C_{\Omega, \mathrm{L}}^{\dagger}\left|0_{\Omega}\right\rangle_{\mathrm{L}}=\left|0_{\Omega}\right\rangle_{\mathrm{I}}^{-}\left|1_{\Omega}\right\rangle_{\mathrm{II}}^{+},
\end{aligned}
$$

and, therefore,

$$
\left|1_{k}\right\rangle_{\mathrm{U}}^{+}=q_{\mathrm{R}}\left|1_{\Omega}\right\rangle_{\mathrm{R}} \otimes\left|0_{\Omega}\right\rangle_{\mathrm{L}}+q_{\mathrm{L}}\left|0_{\Omega}\right\rangle_{\mathrm{R}} \otimes\left|1_{\Omega}\right\rangle_{\mathrm{L}} .
$$

In the short notation, we have introduced the state that reads,

$$
\begin{aligned}
\left|1_{k}\right\rangle_{\mathrm{U}}^{+}= & q_{\mathrm{R}}\left[\cos r_{k}|1000\rangle_{\Omega}-\sin r_{\Omega}|1011\rangle_{\Omega}\right] \\
& +q_{\mathrm{L}}\left[\sin r_{\Omega}|1101\rangle_{\Omega}+\cos r_{\Omega}|0001\rangle_{\Omega}\right] .
\end{aligned}
$$

\section{Fermionic entanglement beyond the single-mode approximation}

Let us now consider the following fermionic maximally entangled state:

$$
|\Psi\rangle=\frac{1}{\sqrt{2}}\left(\left|0_{\omega}\right\rangle_{\mathrm{M}}\left|0_{\Omega}\right\rangle_{\mathrm{U}}+\left|1_{\omega}\right\rangle_{\mathrm{M}}^{+}\left|1_{\Omega}\right\rangle_{\mathrm{U}}^{+}\right),
$$

which is the fermionic analog to Eq. (18), and where the modes labeled with $U$ are Grassman-Unruh modes. To compute the 
Alice-Rob partial density matrix, we trace over region II in in $|\Psi\rangle\langle\Psi|$ and obtain

$$
\begin{aligned}
\rho_{A R}= & \frac{1}{2}\left[C^{4}|000\rangle\langle 000|+S^{2} C^{2}(|010\rangle\langle 010|+| 001\rangle\langle 001|)\right. \\
& +S^{4}|011\rangle\left\langle0 1 1 | + | q _ { \mathrm { R } } | ^ { 2 } \left( C^{2}|110\rangle\langle 110|\right.\right. \\
& \left.+S^{2}|111\rangle\langle 111|\right)+\left|q_{\mathrm{L}}\right|^{2}\left(S^{2}|110\rangle\langle 110|\right. \\
& \left.+C^{2}|100\rangle\langle 100|\right)+q_{\mathrm{R}}^{*}\left(C^{3}|000\rangle\langle 110|\right. \\
& \left.+S^{2} C|001\rangle\langle 111|\right)-q_{\mathrm{L}}^{*}\left(C^{2} S|001\rangle\langle 100|\right. \\
& \left.\left.+S^{3}|011\rangle\langle 110|\right)-q_{\mathrm{R}} q_{\mathrm{L}}^{*} S C|111\rangle\langle 100|\right] \\
& +(\text { H.c. })_{\text {nondiag }},
\end{aligned}
$$

in the basis where $C=\cos r_{\Omega}$ and $S=\sin r_{\Omega}$. To compute the negativity, we first obtain the partial-transpose density matrix (transpose only in the subspace of Alice or Rob) and find its negative eigenvalues. The partial-transpose matrix is block diagonal, and only the following two blocks contribute to negativity,

(1) $\{|100\rangle,|010\rangle,|111\rangle\}$,

$$
\frac{1}{2}\left(\begin{array}{ccc}
C^{2}\left|q_{\mathrm{L}}\right|^{2} & C^{3} q_{\mathrm{R}}^{*} & -q_{\mathrm{R}}^{*} q_{\mathrm{L}} S C \\
C^{3} q_{\mathrm{R}} & S^{2} C^{2} & -q_{\mathrm{L}} S^{3} \\
-q_{\mathrm{R}} q_{\mathrm{L}}^{*} S C & -q_{\mathrm{L}}^{*} S^{3} & \left|q_{\mathrm{R}}\right|^{2} S^{2}
\end{array}\right) .
$$

(2) $\{|000\rangle,|101\rangle,|011\rangle\}$,

$$
\frac{1}{2}\left(\begin{array}{ccc}
C^{4} & -q_{\mathrm{L}} C^{2} S & 0 \\
-q_{\mathrm{L}}^{*} C^{2} S & 0 & q_{\mathrm{R}}^{*} S^{2} C, \\
0 & q_{\mathrm{R}} S^{2} C & S^{4}
\end{array}\right)
$$

where the basis used is $|i j k\rangle=|i\rangle_{\mathrm{M}} \overbrace{|j\rangle_{\mathrm{I}}^{+}|k\rangle_{\mathrm{I}}^{+}}^{\text {Rob }}$. Notice that, although the system is bipartite, the dimension of the partial Hilbert space for Alice is lower than the dimension of the Hilbert space for Rob, which includes particle and antiparticle modes. The eigenvalues only depend on $\left|q_{\mathrm{R}}\right|$ and not on the relative phase between $q_{\mathrm{R}}$ and $q_{\mathrm{L}}$.

The density matrix for the Alice-antiRob modes is obtained by tracing over region $\mathrm{I}$ in $|\Psi\rangle\langle\Psi|$,

$$
\begin{aligned}
\rho_{A \bar{R}}= & \frac{1}{2}\left[C^{4}|000\rangle\langle 000|+S^{2} C^{2}(|010\rangle\langle 010|+| 001\rangle\langle 001|)\right. \\
& +S^{4}|011\rangle\left\langle0 1 1 | + | q _ { \mathrm { R } } | ^ { 2 } \left( C^{2}|100\rangle\langle 100|\right.\right. \\
& \left.+S^{2}|101\rangle\langle 101|\right)+\left|q_{\mathrm{L}}\right|^{2}\left(S^{2}|111\rangle\langle 111|\right. \\
& \left.+C^{2}|101\rangle\langle 101|\right)+q_{\mathrm{L}}^{*}\left(C^{3}|000\rangle\langle 101|\right. \\
& \left.+S^{2} C|010\rangle\langle 111|\right)+q_{\mathrm{R}}^{*}\left(C^{2} S|010\rangle\langle 100|\right. \\
& \left.\left.+S^{3}|011\rangle\langle 101|\right)+q_{\mathrm{R}} q_{\mathrm{L}}^{*} S C|100\rangle\langle 111|\right]+(\text { H.c. })_{\text {nondiag }} .
\end{aligned}
$$

In this case, the blocks of the partial-transpose density matrix, which contribute to the negativity are

(1) $\{|111\rangle,|001\rangle,|100\rangle\}$,

$$
\frac{1}{2}\left(\begin{array}{ccc}
S^{2}\left|q_{\mathrm{L}}\right|^{2} & S^{3} q_{\mathrm{R}}^{*} & q_{\mathrm{R}}^{*} q_{\mathrm{L}} S C \\
S^{3} q_{\mathrm{R}} & C^{2} S^{2} & q_{\mathrm{L}} C^{3} \\
q_{\mathrm{R}} q_{\mathrm{L}}^{*} S C & q_{\mathrm{L}}^{*} C^{3} & \left|q_{\mathrm{R}}\right|^{2} C^{2}
\end{array}\right) .
$$

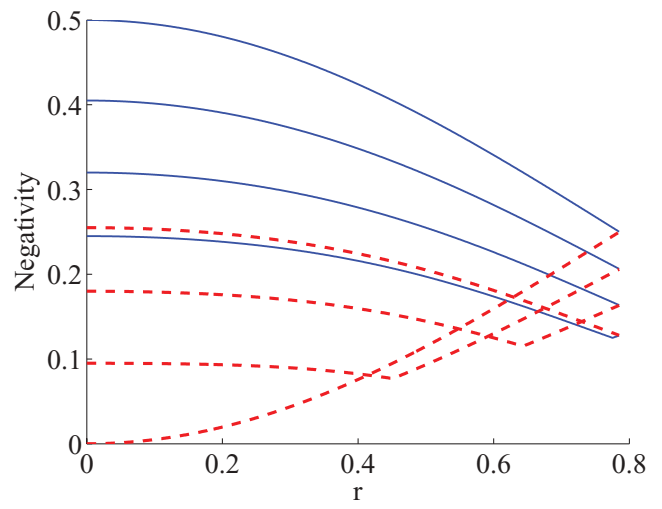

FIG. 3. (Color online) Negativity for the bipartition Alice-Rob (blue continuous) and Alice-antiRob (red dashed) as a function of $r_{\Omega}=\arctan e^{-\pi \Omega_{a} / a}$ for various choices of $\left|q_{\mathrm{R}}\right|$. The blue continuous (red dashed) curves from top to bottom (from bottom to top) correspond to $\left|q_{\mathrm{R}}\right|=1,0.9,0.8,0.7$, respectively. All the curves for Alice-antiRob entanglement have a minimum, except from the extreme case $\left|q_{R}\right|=1$.

(2) $\{|011\rangle,|110\rangle,|000\rangle\}$,

$$
\frac{1}{2}\left(\begin{array}{ccc}
S^{4} & q_{\mathrm{L}} S^{2} C & 0 \\
q_{\mathrm{L}}^{*} S^{2} C & 0 & q_{\mathrm{R}}^{*} C^{2} S \\
0 & q_{\mathrm{R}} C^{2} S & C^{4}
\end{array}\right)
$$

where we have considered the basis $|i j k\rangle=|i\rangle_{\mathrm{M}} \overbrace{|j\rangle_{\mathrm{II}}^{-}|k\rangle_{\mathrm{II}}^{+}}^{\text {Anti-Rob }}$. Once more, the eigenvalues only depend on $\left|q_{\mathrm{R}}\right|$ and not on the relative phase between $q_{\mathrm{R}}$ and $q_{\mathrm{L}}$.

In Fig. 3, we plot the entanglement between Alice-Rob (solid line) and Alice-antiRob (dashed line) modes quantified by the negativity as a function of acceleration for different choices of $\left|q_{\mathrm{R}}\right|$ (in the range $1 \geqslant\left|q_{\mathrm{R}}\right|>1 / \sqrt{2}$ ).

We confirm that the case $\left|q_{\mathrm{R}}\right|=1$ reproduced the results reported in the literature [5]. The entanglement between Alice-Rob modes is degraded as the acceleration parameter increases reaching a nonvanishing minimum value in the infinite-acceleration limit $a \rightarrow \infty$. However, while the entanglement Alice-Rob decreases, entanglement between the Alice-antiRob partition (dashed line) grows. Interestingly, the quantum correlations between the bipartitions Alice-Rob and Alice-antiRob fulfill a conservation law $\mathcal{N}($ Alice-Rob $)+$ $\mathcal{N}($ Alice-antiRob $)=1 / 2$. Note that the choice $\left|q_{\mathrm{R}}\right|=0$ corresponds to an exchange of the Alice-Rob and Alice-antiRob bipartitions. In such cases, the entanglement between Alice and anti-Robs's modes degrades with acceleration, while the entanglement between Alice and Rob's modes grows. In fact, regarding entanglement, the role of the Alice-Rob and Alice-antiRob partitions are exchanged when $\left|q_{\mathrm{R}}\right|<\left|q_{\mathrm{L}}\right|$. This is because there is an explicit symmetry between field excitations in the Rindler wedges. Therefore, we will limit our analysis to $\left|q_{\mathrm{R}}\right|>\left|q_{\mathrm{L}}\right|$.

In the fermionic case, different choices of $\left|q_{\mathrm{R}}\right|$ result in different degrees of entanglement between modes. In particular, the amount of entanglement in the limit of infinite acceleration depends on this choice. Therefore, we can find a special Unruh state, which is more resilient to entanglement degradation. The 
total entanglement is maximal in the infinite-acceleration limit in the case $\left|q_{\mathrm{R}}\right|=1$ (or $\left|q_{\mathrm{L}}\right|=1$ ) in which $\mathcal{N}_{\infty}$ (Alice-Rob) $=$ $\mathcal{N}_{\infty}$ (Alice-antiRob) $=0.25$. In this case, the entanglement lost between Alice-Rob modes is completely compensated by the creation of entanglement between Alice-antiRob modes.

In the case $\left|q_{\mathrm{R}}\right|=\left|q_{\mathrm{L}}\right|=1 / \sqrt{2}$, we see that the behavior of both bipartitions is identical. The entanglement from the inertial perspective is equally distributed between the Alice-Bob and Alice-antiBob partitions and adds up to $\mathcal{N}$ (Alice-Bob) + $\mathcal{N}($ Alice-antiBob $)=0.5$, which corresponds to the total entanglement between Alice-Bob when $\left|q_{\mathrm{R}}\right|=1$. In the infiniteacceleration limit, the case $\left|q_{\mathrm{R}}\right|=\left|q_{\mathrm{L}}\right|$ reaches the minimum total entanglement. To understand this, we note that the entanglement in the Alice-Rob bipartition for $\left|q_{\mathrm{R}}\right|>\left|q_{\mathrm{L}}\right|$ is always monotonic. However, this is not the case for the entanglement between the Alice-antiRob modes. Consider the plot in Fig. 3 for the cases $\left|q_{\mathrm{R}}\right|<1$, for small accelerations, entanglement is degraded in both bipartitions. However, as the acceleration increases, entanglement between Alice-antiRob modes is created compensating the entanglement lost between Alice-Rob. The equilibrium point between degradation and creation is the minimum that Alice-antiRob entanglement curves present. Therefore, if $\left|q_{R}\right|<1$, the entanglement lost is not entirely compensated by the creation of entanglement between Alice-antiRob resulting in a less entangled state in the infinite-acceleration limit.

In the case $\left|q_{\mathrm{R}}\right|=\left|q_{\mathrm{L}}\right|=1 / \sqrt{2}$, entanglement is always degraded between Alice-antiRob modes resulting in the state, among all the possible elections of Unruh modes, with less entanglement in the infinite-acceleration limit.

\section{CONCLUSIONS}

We have shown that the single-mode approximation used in the relativistic quantum-information literature, especially to analyze entanglement between field modes from the perspective of observers in uniform acceleration, does not hold for general states. The single-mode approximation attempts to relate a single Minkowski frequency mode (observed by inertial observers) with a single Rindler frequency mode (observed by uniformly accelerated observers).

We show that the state canonically analyzed in the literature corresponds to a maximally entangled state of a Minkowski mode and a specific kind of Unruh mode (not two Minkowski modes). We analyze the entanglement between two bosonic or fermionic modes in the case when, from the inertial perspective, the state corresponds to a maximally entangled state between a Minkowski frequency mode and an arbitrary Unruh frequency mode.
We find that the entanglement between modes described by an Unruh observer and a Rindler observer constrained to move in Rindler region I (Alice-Rob) are always degraded with acceleration. In the bosonic case, the entanglement between the inertial modes and region II Rindler modes (Alice-antiRob) are also degraded with acceleration. We find that, in this case, the rate of entanglement degradation is independent of the choice of Unruh modes.

For the fermionic case, the entanglement between the inertial and region I Rindler modes (Alice-Rob) is degraded as the acceleration increases reaching a minimum value when it tends to infinity. Therefore, there is entanglement survival in the limit of infinite acceleration for any choice of Unruh modes. However, we find an important difference with the bosonic case: The amount of surviving entanglement depends on the specific election of such modes.

We also find that the entanglement between inertial and region II Rindler modes (Alice-antiRob) can be created and degraded depending on the election of Unruh modes. This gives rise to different values of entanglement in the infiniteacceleration limit. Interestingly, in the fermionic case, one can find a state, which is most resilient to entanglement degradation. This corresponds to the Unruh mode with $\left|q_{\mathrm{R}}\right|=$ 1 , which is the Unruh mode considered in the canonical studies of entanglement $[1,4,5,15,21,22]$. It could be argued that this is the most natural choice of Unruh modes, since, for this choice $\left(\left|q_{R}\right|=1\right)$, the entanglement for very small accelerations $(a \rightarrow 0)$ is mainly contained in the subsystem Alice-Rob. In this case, there is nearly no entanglement between the Alice-antiRob modes. However, other choices of Unruh modes become relevant if one wishes to consider an entangled state from the inertial perspective, which involves only Minkowski frequencies. We have shown that a Minkowski wave packet involving a superposition of general Unruh modes can be constructed in such a way that the corresponding Rindler state involves a single frequency. This result is especially interesting, since it presents an instance where the single-mode approximation can be considered recovering the standard results in the literature.

\section{ACKNOWLEDGMENTS}

We thank R. B. Mann, J. Leon, B. L. Hu, P. Alsing, T. Ralph, T. Downes, and K. Pachucki for interesting discussions and useful comments. I.F. was supported by EPSRC [CAF Grant No. EP/G00496X/2]. J.L. was supported in part by STFC (UK) Rolling Grant No. PP/D507358/1. E.M.-M. was supported by a CSIC JAE-PREDOC2007 Grant and by the Spanish MICINN Project No. FIS2008-05705/FIS.
[1] P. M. Alsing and G. J. Milburn, Phys. Rev. Lett. 91, 180404 (2003).

[2] H. Terashima and M. Ueda, Phys. Rev. A 69, 032113 (2004).

[3] Y. Shi, Phys. Rev. D 70, 105001 (2004).

[4] I. Fuentes-Schuller and R. B. Mann, Phys. Rev. Lett. 95, 120404 (2005).
[5] P. M. Alsing, I. Fuentes-Schuller, R. B. Mann, and T. E. Tessier, Phys. Rev. A 74, 032326 (2006).

[6] J. L. Ball, I. Fuentes-Schuller, and F. P. Schuller, Phys. Lett. A 359, 550 (2006).

[7] G. Adesso, I. Fuentes-Schuller, and M. Ericsson, Phys. Rev. A 76, 062112 (2007). 
[8] K. Brádler, Phys. Rev. A 75, 022311 (2007).

[9] Y. Ling, S. He, W. Qiu, and H. Zhang, J. Phys. A 40, 9025 (2007).

[10] D. Ahn, Y. Moon, R. Mann, and I. Fuentes-Schuller, J. High Energy Phys. 08 (2008) 062.

[11] Q. Pan and J. Jing, Phys. Rev. D 78, 065015 (2008).

[12] P. M. Alsing, D. McMahon, and G. J. Milburn, J. Opt. B: Quantum Semiclass. Opt. 6, S834 (2004).

[13] J. Doukas and L. C. L. Hollenberg, Phys. Rev. A 79, 052109 (2009).

[14] G. VerSteeg and N. C. Menicucci, Phys. Rev. D 79, 044027 (2009).

[15] J. León and E. Martín-Martínez, Phys. Rev. A 80, 012314 (2009).

[16] G. Adesso and I. Fuentes-Schuller, Quantum Inf. Comput. 9, 0657 (2009).

[17] A. Datta, Phys. Rev. A 80, 052304 (2009).

[18] S.-Y. Lin and B. L. Hu, Phys. Rev. D 81, 045019 (2010).

[19] J. Wang, J. Deng, and J. Jing, Phys. Rev. A 81, 052120 (2010).

[20] E. Martín-Martínez, L. J. Garay, and J. León, Phys. Rev. D 82, 064006 (2010).

[21] E. Martín-Martínez and J. León, Phys. Rev. A 80, 042318 (2009).

[22] E. Martín-Martínez and J. León, Phys. Rev. A 81, 032320 (2010).

[23] J. L. Ball, I. Fuentes-Schuller, and F. P. Schuller, Phys. Lett. A 359, 550 (2006).

[24] I. Fuentes, R. B. Mann, E. Martín-Martínez, and S. Moradi, Phys. Rev. D 82, 045030 (2010).

[25] E. Martín-Martínez, L. J. Garay, and J. León, Phys. Rev. D 82, 064028 (2010).
[26] K. Brádler, Phys. Rev. A 75, 022311 (2007).

[27] X.-H. Ge and S. P. Kim, Class. Quantum Grav. 25, 075011 (2008).

[28] Q. Pan and J. Jing, Phys. Rev. A 77, 024302 (2008).

[29] Q. Pan and J. Jing, Phys. Rev. D 78, 065015 (2008).

[30] S. Moradi, Phys. Rev. A 79, 064301 (2009).

[31] A. G. S. Landulfo and G. E. A. Matsas, Phys. Rev. A 80, 032315 (2009).

[32] D. C. M. Ostapchuk and R. B. Mann, Phys. Rev. A 79, 042333 (2009).

[33] E. Martín-Martínez and J. León, Phys. Rev. A 81, 052305 (2010).

[34] N. B. Narozhny, A. M. Fedotov, B. M. Karnakov, V. D. Mur, and V. A. Belinskii, Phys. Rev. D 65, 025004 (2001).

[35] S. A. Fulling and W. G. Unruh, Phys. Rev. D 70, 048701 (2004).

[36] N. B. Narozhny, A. M. Fedotov, B. M. Karnakov, V. D. Mur, and V. A. Belinskii, Phys. Rev. D 70, 048702 (2004).

[37] S. Takagi, Prog. Theor. Phys. Suppl. 88, 1 (1986).

[38] W. G. Unruh, Phys. Rev. D 14, 870 (1976).

[39] N. D. Birrell and P. C. W. Davies, Quantum Fields in Curved Space (Cambridge University Press, Cambridge, UK, 1984).

[40] National Institute of Standards and Technology, Digital Library of Mathematical Functions. 2010-05-07 [http://dlmf.nist.gov/].

[41] R. Jáuregui, M. Torres, and S. Hacyan, Phys. Rev. D 43, 3979 (1991).

[42] P. Langlois, Phys. Rev. D 70, 104008 (2004).

[43] Z. Jianyang and L. Zhijian, Int. J. Theor. Phys. 38, 575 (1999). 